\title{
Utilization and prescription patterns of traditional Chinese medicine for patients with hepatitis $C$ in Taiwan: a population- based study
}

Chia-Yu Liu ${ }^{1,2,3}$, Jui-Ying Chu ${ }^{2}$, Jen-Huai Chiang ${ }^{4,5,6}$, Hung-Rong Yen ${ }^{4,7,8^{*+}}$ and Chung-Hua Hsu ${ }^{1,2,8^{*}+}$

\begin{abstract}
Background: To characterize the utilization of Traditional Chinese Medicine (TCM) among patients with hepatitis C (HC). Methods: This study examined datasets from the National Health Insurance Research Database in Taiwan. One cohort, including one million patients randomly sampled from the beneficiaries of the National Health Insurance Programme from January 1 to December 31 in 2010, was chosen for this analysis. People who had at least three outpatient or inpatient records and had been diagnosed with hepatitis C virus infection from 2000 to 2010 were defined as patients with HC. Patients with HC who had at least one TCM outpatient clinical record from 2000 to 2010 were defined as TCM users $(N=5,691)$, whereas patients with no TCM outpatient records were defined as non-TCM users $(N=2,876)$. The demographic data, treatment modalities and disease distributions of TCM users were analysed.
\end{abstract}

Results: Overall, $66.4 \%$ of the patients with HC had used TCM from 2000 to 2010. Of the TCM users, $54.1 \%$ were female. The utilization rate of TCM increased with age and peaked in the age group of those $40-64$ years old. Herbal remedies (52.4\%) were the most commonly used agents, followed by combination therapy (46.4\%) and acupuncture alone (1.2\%). Patients who had more extrahepatic diseases and were taking more antiviral agents tended to visit TCM clinics. Jia-Wei-Xiao-Yao-San and Dan-Shen (Salvia miltiorrhiza) were the most commonly used formula and single herb, with 88,124 person-days and 59,252 person-days, respectively.

Conclusions: Our nationwide population-based study revealed a high prevalence and specific usage patterns of TCM in patients with HC in Taiwan.

Keywords: Complementary and alternative medicine, Hepatitis C, National Health Insurance Research Database, Traditional Chinese medicine

\section{Background}

The use of complementary and alternative medicine (CAM) is expanding throughout the world $[1,2]$. According to the World Health Organization (W.H.O.), CAM includes Ayurveda, traditional Chinese medicine (TCM) and Unani medicine. TCM is widely used in East Asia [3]. Of the twenty-three million people in Taiwan,

\footnotetext{
* Correspondence: hungrongyen@gmail.com; owlherbs@yahoo.com.tw ${ }^{\dagger}$ Equal contributors

${ }^{4}$ Research Centre for Chinese Medicine \& Acupuncture, China Medical University, Taichung, Taiwan

'Institute of Traditional Medicine, School of Medicine, National Yang-Ming University, Taipei, Taiwan

Full list of author information is available at the end of the article
}

$29.1 \%$ used TCM to treat disease in 2014, whereas up to three-fourths of South Korean adults utilized TCM to treat a specific disease [4].

Hepatitis C virus (HCV), which affects 180 million people globally, is a leading cause of chronic hepatitis, cirrhosis, and hepatocellular carcinoma [5]. Conventional anti-viral therapy consisting of pegylated interferon and ribavirin is associated with many intolerable side effects and low response rates in some patients' genotypes [6]. Therefore, patients often seek for alternative treatments to promote healing and obtain support [7]. However, the communication regarding CAM between patients and physicians is relatively poor $[8,9]$, and the 
non-disclosure rate is higher than $70 \%$. Many physicians feel uncomfortable discussing CAM because of their limited knowledge of the subject. Patients avoid discussing CAM with their doctors because they fear receiving a negative response [10]. To provide holistic care to patients with hepatitis $\mathrm{C}(\mathrm{HC})$, physicians should understand the approaches used by patients for symptom relief and health maintenance. In the United States, 80 \% of patients with $\mathrm{HC}$ used CAM according to a report from 2007 [11]. Compared with patients with fatty liver disease, patients with $\mathrm{HC}$ were approximately 3 times more likely to use CAM [12]. Because there has been growing interest in using CAM to treat populations with $\mathrm{HC}$ worldwide [13], information about CAM and comprehensive studies on its prevalence, usage patterns, efficacy and safety are important.

TCM, defined by the National Centre for Complementary and Integrative Health (NCCIH, U.S.A.) as an entire medical system of CAM, is a well-established medical system that has been used for more than 2,000 years. TCM is commonly used by the Chinese population, as well as by those in many other countries [14], including Taiwan [15]. In Taiwan, TCM has been reimbursed by the National Health Insurance (NHI) programme since 1996. As of 2014, $99.9 \%$ of Taiwan's population were enrolled in the NHI. All of the claims data have been collected in the National Health Insurance Research Database (NHIRD). According to the NHI programme guidelines, TCMs are only provided for outpatient care including Chinese herbal prescriptions, acupuncture, and traumatology manipulative therapy. The utilization prevalence of TCM in Taiwan ranges from $19.8 \%$ to $77.9 \%$ for many diseases including colon cancer [16], liver cancer [17], osteoporosis [18], and type II diabetes mellitus [19]. However, the utilization and prescription patterns of TCM in $\mathrm{HC}$ are lacking.

To characterize the utilization patterns and trends in TCM usage among patients with $\mathrm{HC}$, we analysed a cohort of one million randomly sampled beneficiaries from the NHIRD in 2010. The results of this study should provide valuable information for physicians and for patients with $\mathrm{HC}$.

\section{Methods}

\section{Data source}

As previously described in detail [20], all TCM services covered under the NHI are provided only in ambulatory clinics. In Taiwan, TCM physicians (those who have received a series of training in Chinese or both Chinese and Western medicine, all of whom must pass national licensing examinations and complete residency training programmes in hospitals) are requested to make diagnoses based on the International Classification of Disease, 9th Revision, Clinical Modification (ICD-9-CM) coding [16].
This study chose one cohort for the analysis, which included one million patients randomly sampled from the beneficiaries of the NHI programme in 2010. The NHIRD contains information on the medical care facilities, physician specialties, and patients' gender, dates of birth, dates of visit, masked identification numbers, prescriptions, management and diagnosis codes in the ICD-9-CM. A maximum of three diagnostic codes were listed in the NHIRD, and all the diagnoses were analysed in our study. This study was approved by the Research Ethics Committee of the Taipei City Hospital (TCHIRB-10406112-E).

\section{Study subjects}

The study subjects were selected from a random sample of one million individuals in 2010 in the following manner (Fig. 1): People who had at least three outpatient or inpatient records and had been diagnosed with HCV from January 1, 2000, to December 31, 2010, were defined as patients with HC (ICD-9-CM codes 07054, 0707, 07041, 07044, 07051, V0262). Based on this criteria, there were 8,567 patients older than 18 years old with a new $\mathrm{HCV}$ infection diagnosed starting from the index date of January 1, 2000. Patients with $\mathrm{HC}$ who had at least one TCM clinical record from 2000 to 2010 were defined as TCM users $(N=5,691)$, whereas those who had no TCM records during the same period were defined as non-TCM users $(N=2,876)$. All study subjects were followed until December 31, 2011.

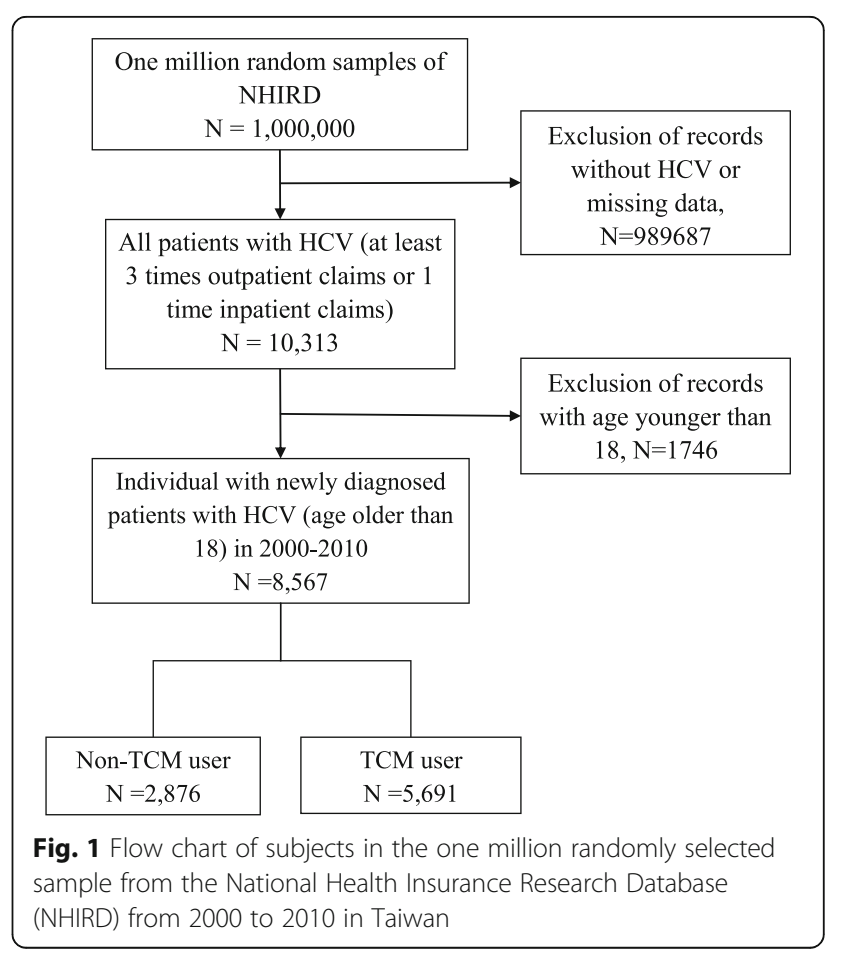




\section{Statistical analysis}

The data were analysed using SAS software program, version 9.4 (SAS Institute Inc., Cary, NC, U.S.A.). A univariate analysis was used to compare the TCM users with the non-TCM users. Chi-squared test was performed to assess the relationships between the categorical variables and to examine the differences between TCM users and non-users. Person-years for the follow-up period were calculated for each patient until diagnosis of multisite diseases, censor or December 31, 2011. The person-years and overlapping confidence interval (CI) were calculated to assess incidence density rates. To compare the study cohort to the comparison cohort rate, ratios were examined using a Poisson regression model. Moreover, we estimated the adjusted hazard ratios using Cox proportional hazards model. A $P$ value $<0.05$ was considered statistically significant.

\section{Results}

Demographic characteristics of the TCM users with HC

Of the patients with $\mathrm{HC}, 66.4 \%(N=5,691)$ had previously used TCM (Table 1). In the TCM users, $54.1 \%$ were female, and this differed from the percentage in non-TCM users, which was only $39.9 \%$. In both TCM users and non-users, the highest proportion of patients was in the age group from 40 to 64 years old. Regarding the comorbidities of patients with $\mathrm{HC}$, the incidence of most diseases, including osteoarthritis, sicca syndrome, thyroid disorders, chronic obstructive pulmonary disease, and hepatitis $\mathrm{B}$, were higher in TCM users, except for diabetes mellitus, which had a similar incidence in both groups. In contrast, the incidence of hepatocellular carcinoma, liver cirrhosis, and alcohol-related diseases were higher in non-TCM users.

To examine the frequency of utilization of Western medicine, we analysed the claims data according to the antiviral or hepatoprotective agents used (Table 1). A higher percentage of TCM users than non-TCM users had ever used these agents to control hepatitis C (all $p$-values $<0.001$ ). TCM users tended to use interferon-alpha, ribavirin, and silymarin to treat $\mathrm{HCV}(17.1 \%, 17.1 \%$, and $73.9 \%$, respectively).

With regard to TCM outpatient visits (Table 2), herbal remedies $(52.4 \%)$ were the most commonly used therapeutic approach, followed by combined therapy (46.4\%) and acupuncture alone (1.12\%). As for the frequency of visits, the majority of patients visited TCM clinics fewer than three times (74.01\%). Only $16.64 \%$ of the patients visited TCM clinics more than six times.
Table 1 Demographic characteristics of the patients with hepatitis C in Taiwan in 2000-2011

\begin{tabular}{|c|c|c|c|c|c|}
\hline \multirow[t]{3}{*}{ Variable } & \multirow{2}{*}{\multicolumn{2}{|c|}{$\begin{array}{l}\text { Non-TCM } \\
N=2876 \\
(33.57 \%)\end{array}$}} & \multirow{2}{*}{\multicolumn{2}{|c|}{$\begin{array}{l}\text { TCM } \\
N=5691 \\
(66.43 \%)\end{array}$}} & \multirow[t]{3}{*}{$p$ value } \\
\hline & & & & & \\
\hline & $n$ & $\%$ & $n$ & $\%$ & \\
\hline \multicolumn{5}{|l|}{ Sex } & \multirow[t]{3}{*}{$<0.0001$} \\
\hline Female & 1148 & 39.92 & 3081 & 54.14 & \\
\hline Male & 1728 & 60.08 & 2610 & 45.86 & \\
\hline \multicolumn{5}{|l|}{ Age at baseline } & \multirow[t]{5}{*}{$<0.0001$} \\
\hline $18-39$ & 364 & 12.66 & 945 & 16.61 & \\
\hline $40-64$ & 1451 & 50.45 & 3395 & 59.66 & \\
\hline$\geq 65$ & 1061 & 36.89 & 1351 & 23.74 & \\
\hline Mean (STD) & 58.34 & 14.85 & 53.92 & 13.97 & \\
\hline \multicolumn{5}{|l|}{ Urbanization $^{a}$} & \multirow[t]{5}{*}{$<0.0001$} \\
\hline 1 (highest) & 574 & 19.96 & 1215 & 21.35 & \\
\hline 2 & 731 & 25.42 & 1652 & 29.03 & \\
\hline 3 & 447 & 15.54 & 939 & 16.50 & \\
\hline $4+$ (lowest) & 1124 & 39.08 & 1885 & 33.12 & \\
\hline \multicolumn{6}{|l|}{ Co-morbidity } \\
\hline Hepatitis B & 570 & 19.82 & 1356 & 23.83 & $<0.0001$ \\
\hline Liver cirrhosis & 989 & 34.39 & 1618 & 28.43 & $<0.0001$ \\
\hline hepatocellular carcinoma & 535 & 18.60 & 898 & 15.78 & 0.0009 \\
\hline Alcohol-related disease & 294 & 10.22 & 477 & 8.38 & 0.0049 \\
\hline $\begin{array}{l}\text { Chronic obstructive pulmonary } \\
\text { disease }\end{array}$ & 508 & 17.66 & 1402 & 24.64 & $<0.0001$ \\
\hline Diabetes Mellitus & 1046 & 36.37 & 2143 & 37.66 & 0.2449 \\
\hline Thyroid disorders & 145 & 5.04 & 631 & 11.09 & $<0.0001$ \\
\hline Rheumatoid arthritis & 89 & 3.09 & 400 & 7.03 & $<0.0001$ \\
\hline Osteoarthritis & 953 & 33.14 & 2922 & 51.34 & $<0.0001$ \\
\hline Depression & 174 & 6.05 & 650 & 11.42 & $<0.0001$ \\
\hline Sicca syndrome & 78 & 2.71 & 423 & 7.43 & $<0.0001$ \\
\hline \multicolumn{5}{|l|}{ Charlson comorbidity index score } & \multirow[t]{4}{*}{$<0.0001$} \\
\hline 0 & 1203 & 41.83 & 3024 & 53.14 & \\
\hline 1 & 377 & 13.11 & 769 & 13.51 & \\
\hline More than 2 & 1296 & 45.06 & 1898 & 33.35 & \\
\hline \multicolumn{6}{|l|}{ Anti-viral or hepatoprotective agents } \\
\hline Interferon alfa & 392 & 13.63 & 974 & 17.11 & $<0.0001$ \\
\hline Ribavirin & 391 & 13.60 & 973 & 17.10 & $<0.0001$ \\
\hline Silymarin & 1916 & 66.62 & 4204 & 73.87 & $<0.0001$ \\
\hline Times of visits (mean, SD) & 15.61 & 17.18 & 21.23 & 23.18 & $<0.0001$ \\
\hline
\end{tabular}

${ }^{a}$ The townships within which subjects registered for insurance were grouped into 4 levels of urbanization, based on a score calculated by incorporating variables indicating population density (people $/ \mathrm{km} 2$ ), and population ratio of different educational levels, population ratio of elderly, population ratio of people of agriculture workers and the number of physicians per 100,000 people 
Table 2 Distribution of Chinese medicine according to type of Chinese Medicine treatment received in patients with hepatitis C, stratified by number of outpatients visits

\begin{tabular}{lllll}
\hline $\begin{array}{l}\text { Number of TCM } \\
\text { visits (times/per year) }\end{array}$ & Only Chinese herbal remedies & Only Acupuncture or traumatology & $\begin{array}{l}\text { Combination both treatment } \\
N=2643(46.44 \%)\end{array}$ & $\begin{array}{l}\text { Total of TCM } \\
(N=5691)\end{array}$ \\
\hline $1-3$ & $2423(81.20 \%)$ & $N=64(1.12 \%)$ & $1725(65.27 \%)$ & $4212(74.01 \%)$ \\
$4-6$ & $212(7.10 \%)$ & $64(100 \%)$ & $320(12.11 \%)$ & $532(9.35 \%)$ \\
$>6$ & $349(11.70 \%)$ & 0 & $598(22.63 \%)$ & $947(16.64 \%)$ \\
\hline
\end{tabular}

The prevalence of hepatitis B, liver cirrhosis, alcoholrelated disease, chronic obstructive pulmonary disease and diabetes mellitus was lower in TCM users than non-TCM users in Taiwanese HC patients

After adjusting for the frequency of outpatient and inpatient visits, Charlson comorbidity index score, and type of service used - only herbal medicine, only acupuncture or a combination - we calculated the disease hazard ratio (HR) of TCM users versus non-TCM users to compare the prevalence of comorbidities between them. We found that TCM users tended to have certain comorbidities less frequently than non-TCM users (Table 3). TCM users had a 0.55 times lower HR of having hepatitis B than non-TCM users (95\% confidence interval 0.46 - 0.66). Patients who received TCM treatment also had a lower ratio for liver cirrhosis (HR 0.42, with $95 \%$ CI 0.37 - 0.48) and alcohol-related disease (HR: 0.31 for the $40-64$ age group and 0.41 overall). The prevalence of chronic obstructive pulmonary disease was also lower in the TCM users (HR: 0.59 overall). Diabetes mellitus also showed a lower incidence in TCM users than non-TCM users overall and in each age group (HR: $0.48,0.40,0.46$ and 0.64 for the sample overall and for the $<40,40-64$, and $>60$ age groups, respectively). However, after adjusting for times of visit, Charlson comorbidity index score, and type of TCM service used, the hazard ratio of comorbidities such as sicca syndrome, rheumatoid arthritis, depression, and thyroid disorders was less than 1 , with no significance.

\section{Frequency distribution of disease categories in TCM versus non-TCM visits}

To delineate the frequency distributions of the disease categories (as the reasons for visits) for the TCM and non-TCM visits, we analysed the ICD-9-CM codes from the claims data (Table 4). There was a significant difference in the disease distributions between the TCM and non-TCM users $(P<0.0001)$. Among all of the visits, infectious diseases (99.68\%), which included viral hepatitis, were the most common reasons that TCM users visited TCM clinics. Digestive system diseases (99.61\%), which included chronic liver disease, were the second most common reason that TCM users visited TCM clinics. Symptoms/signs and ill-defined conditions (97.96 \%) and respiratory system diseases (97.93 \%) accounted for the third and fourth disease categories, respectively, followed by diseases of the musculoskeletal system and connective tissue (93.89\%) and injuries (90.77 \%). For non-TCM users, infectious diseases $(99.51 \%)$ were the most common reason for visiting Western medical clinics, followed by digestive system diseases (97.25\%) and symptoms/signs and ill-defined conditions (89.33\%). When TCM users required medical services, their utilization patterns were similar to those of non-TCM users.

\section{The most commonly used TCM prescriptions}

To comprehensively understand the TCM prescriptions, including the formulas and herbs, we analysed the claims data, and the results are shown in Table 5. Of the 10 most common formulas of TCM used by patients with HC, Jiawei-xiao-yao-san $(88,124$ person-days) was the most commonly used. Xiao-chai-hu-tang (39,837 person-days) and Long-dan-xie-gan-tang (36,293 person-days) accounted for the second and third most commonly used formulas, respectively. With regard to the single herbs used for TCM by patients with $\mathrm{HC}$, Dan-shen (59,252 person-days) was the most common. Yan-hu-suo (41,875 person-days) and Huang-qin (35,273 person-days) were the second and third most commonly used herbs, respectively.

\section{Discussion}

This research is the first large-scale study on the utilization patterns of TCM by patients with $\mathrm{HC}$ and was conducted by analysing claims data from TCM and non-TCM clinic visits covered by the NHI in Taiwan. In a previous study [21], Chen et al. investigated the frequency and prescription patterns of Chinese herbal medicine for chronic hepatitis, including viral hepatitis and alcoholic hepatitis, and revealed the same three most common herbal formulas as in our study and a similar age group of patients, approximately in their 40s to 50s, seeking TCM. However, this study focused only on hepatitis $\mathrm{C}$ and demonstrated a different gender predominance and more details on comorbidities. According to Sievert's review [22], the prevalence rate of hepatitis $\mathrm{C}$ is as high as $4 \%$, but the diagnosis rate is only $1.3 \%$, which is similar to our report. This discrepancy may be due to the fact that only symptomatic patients with $\mathrm{HC}$ would visit the hospital and have 
Table 3 Incidence rate ratio for common disease between non-TCM and TCM user in different age groups

\begin{tabular}{|c|c|c|c|c|c|c|c|c|}
\hline \multirow{2}{*}{$\begin{array}{l}\text { Age groups (year-old) } \\
\text { Disease (ICD-9-CM) }\end{array}$} & \multicolumn{3}{|c|}{ Non-TCM user } & \multicolumn{3}{|l|}{ TCM user } & \multirow[t]{2}{*}{ IRR $(95 \%$ Cl) } & \multirow{2}{*}{$\begin{array}{l}\text { Adjusted HR } \\
(95 \% \mathrm{Cl})\end{array}$} \\
\hline & $\overline{N(\%)}$ & Person-years & $\mathbb{I R}^{a}$ & $\overline{N(\%)}$ & Person-years & $\mathbb{R}^{a}$ & & \\
\hline \multicolumn{9}{|c|}{ Hepatitis B $(70.2,070.3$, V02.61) } \\
\hline All & $570(19.82)$ & 13085 & 43.56 & $1356(23.83)$ & 39350 & 34.46 & $0.79(0.72-0.87)^{* * *}$ & $0.55(0.46-0.66)^{* * *}$ \\
\hline $18-39$ & $88(24.18)$ & 1901 & 46.3 & $239(25.29)$ & 7189 & 33.24 & $0.72(0.56-0.92)^{* *}$ & $0.41(0.25-0.69)^{* * *}$ \\
\hline $40-64$ & $327(22.54)$ & 6923 & 47.23 & $842(24.80)$ & 23900 & 35.23 & $0.75(0.66-0.85)^{* * *}$ & $0.48(0.37-0.6)^{* * *}$ \\
\hline$\geq 65$ & 155(14.61) & 4261 & 36.37 & $275(20.36)$ & 8260 & 33.29 & $0.92(0.75-1.11)$ & $0.82(0.58-1.17)$ \\
\hline \multicolumn{9}{|c|}{ Liver cirrhosis $(571.2,571.5,571.6,572.2,572.3,572.4,572.8,573.0)$} \\
\hline All & 989(34.39) & 13085 & 75.58 & $1618(28.43)$ & 39350 & 41.12 & $0.54(0.50-0.59)^{* * *}$ & $0.42(0.37-0.48)^{* * *}$ \\
\hline $18-39$ & $45(12.36)$ & 1901 & 23.68 & 108(11.43) & 7189 & 15.02 & $0.63(0.45-0.90)^{*}$ & $0.43(0.25-0.73)^{* *}$ \\
\hline $40-64$ & 489(33.70) & 6923 & 70.63 & $951(28.01)$ & 23900 & 39.79 & $0.56(0.51-0.63)^{* * *}$ & $0.39(0.33-0.46)^{* * *}$ \\
\hline$\geq 65$ & 455(42.88) & 4261 & 106.77 & $559(41.38)$ & 8260 & 67.67 & $0.63(0.56-0.72)^{* * *}$ & $0.54(0.44-0.66)^{* * *}$ \\
\hline \multicolumn{9}{|c|}{ hepatocellular carcinoma (155) } \\
\hline All & $535(18.60)$ & 13085 & 40.89 & $898(15.78)$ & 39350 & 22.82 & $0.56(0.50-0.62)^{* * *}$ & $0.78(0.45-1.36)$ \\
\hline $18-39$ & $6(1.65)$ & 1901 & 3.16 & $33(3.49)$ & 7189 & 4.59 & $1.45(0.61-3.47)$ & $1.25(0.12-12.75)$ \\
\hline $40-64$ & $242(16.68)$ & 6923 & 34.96 & $514(15.14)$ & 23900 & 21.51 & $0.62(0.53-0.72)^{* * *}$ & $0.98(0.43-2.21)$ \\
\hline$\geq 65$ & $287(27.05)$ & 4261 & 67.35 & $351(25.98)$ & 8260 & 42.49 & $0.63(0.54-0.74)^{* * *}$ & $0.72(0.29-1.75)$ \\
\hline
\end{tabular}

Alcohol-related disease (291, 303.0, 303.9, 305.0, 571.0, 571.1, 571.3)

$\begin{array}{lllllllll}\text { All } & 294(10.22) & 13085 & 22.47 & 477(8.38) & 39350 & 12.12 & 0.54(0.47-0.62)^{* * *} & 0.41(0.32-0.51)^{* * *} \\ 18-39 & 65(17.86) & 1901 & 34.2 & 124(13.12) & 7189 & 17.25 & 0.50(0.37-0.68)^{* * *} & 0.37(0.24-0.58)^{* * *} \\ 40-64 & 185(12.75) & 6923 & 26.72 & 290(8.54) & 23900 & 12.13 & 0.45(0.38-0.55)^{* * *} & 0.31(0.23-0.42)^{* * *} \\ \geq 65 & 44(4.15) & 4261 & 10.33 & 63(4.66) & 8260 & 7.63 & 0.74(0.50-1.09) & 0.61(0.33-1.16)\end{array}$

Chronic obstructive pulmonary disease $(491,492)$

$\begin{array}{lllllllll}\text { All } & 508(17.66) & 13085 & 38.82 & 1402(24.64) & 39350 & 35.63 & 0.92(0.83-1.12) & 0.59(0.50-0.70)^{* * *} \\ 18-39 & 27(7.42) & 1901 & 14.21 & 120(12.70) & 7189 & 16.69 & 1.18(0.77-1.78) & 0.94(0.45-1.97) \\ 40-64 & 198(13.65) & 6923 & 28.6 & 786(23.15) & 23900 & 32.89 & 1.15(0.98-1.34) & 0.67(0.52-0.86)^{* *} \\ \geq 65 & 283(26.67) & 4261 & 66.41 & 496(36.71) & 8260 & 60.05 & 0.90(0.78-1.05) & 0.69(0.53-0.89)^{* *}\end{array}$

Diabetes Mellitus (250)

$\begin{array}{lll}\text { All } & 1046(36.37) & 13085 \\ 18-39 & 55(15.11) & 1901 \\ 40-64 & 572(39.42) & 6923 \\ \geq 65 & 419(39.49) & 4261\end{array}$

$\begin{array}{lll}79.94 & 2143(37.66) & 39350 \\ 28.94 & 151(15.98) & 7189 \\ 82.62 & 1358(40.00) & 23900 \\ 98.32 & 634(46.93) & 8260\end{array}$

$\begin{array}{lll}54.46 & 0.68(0.63-0.73)^{* * *} & 0.48(0.42-0.55)^{* * *} \\ 21 & 0.73(0.53-0.99)^{*} & 0.40(0.22-0.71)^{* *} \\ 56.82 & 0.69(0.62-0.76)^{* * *} & 0.46(0.38-0.54)^{* * *} \\ 76.75 & 0.78(0.69-0.88)^{* * *} & 0.64(0.51-0.8)^{* * *}\end{array}$

Thyroid disease $(240,241,242,244)$

$\begin{array}{lllllllll}\text { All } & 145(5.04) & 13085 & 11.08 & 631(11.09) & 39350 & 16.04 & 1.44(1.21-1.73)^{* * *} & 0.90(0.67-1.21) \\ 18-39 & 21(5.77) & 1901 & 11.05 & 105(11.11) & 7189 & 14.6 & 1.32(0.83-2.11) & 1.33(0.54-3.28) \\ 40-64 & 79(5.44) & 6923 & 11.41 & 418(12.31) & 23900 & 17.49 & 1.53(1.21-1.95)^{* * *} & 0.9(0.6-1.36) \\ \geq 65 & 45(4.24) & 4261 & 10.56 & 108(7.99) & 8260 & 13.07 & 1.24(0.87-1.75) & 0.92(0.53-1.58) \\ \text { Rheumatoid arthritis (714) } & & & & & & & & \\ \text { All } & 89(3.09) & 13085 & 6.8 & 400(7.03) & 39350 & 10.17 & 1.49(1.19-1.88)^{* * *} & 1.18(0.82-1.69) \\ 18-39 & 11(3.02) & 1901 & 5.79 & 42(4.44) & 7189 & 5.84 & 1.01(0.52-1.96) & 0.88(0.28-2.78) \\ 40-64 & 45(3.10) & 6923 & 6.5 & 266(7.84) & 23900 & 11.13 & 1.71(1.25-2.35)^{* * *} & 1.38(0.81-2.36) \\ \geq 65 & 33(3.11) & 4261 & 7.74 & 92(6.81) & 8260 & 11.14 & 1.44(0.97-2.14) & 1.24(0.69-2.22)\end{array}$


Table 3 Incidence rate ratio for common disease between non-TCM and TCM user in different age groups (Continued)

\begin{tabular}{|c|c|c|c|c|c|c|c|c|}
\hline \multicolumn{9}{|c|}{ Osteoarthritis (715) } \\
\hline All & 953(33.14) & 13085 & 72.83 & 2922(51.34) & 39350 & 74.26 & $1.02(0.95-1.10)$ & $0.75(0.66-0.86)^{* * *}$ \\
\hline $18-39$ & $46(12.64)$ & 1901 & 24.2 & $215(22.75)$ & 7189 & 29.91 & $1.24(0.90-1.70)$ & $1.2(0.64-2.27)$ \\
\hline $40-64$ & $427(29.43)$ & 6923 & 61.68 & $1777(52.34)$ & 23900 & 74.35 & $1.21(1.08-1.34)^{* * *}$ & $0.85(0.71-1.03)$ \\
\hline$\geq 65$ & $480(45.24)$ & 4261 & 112.64 & $930(68.84)$ & 8260 & 112.59 & $1.00(0.90-1.12)$ & $0.82(0.67-1)$ \\
\hline \multicolumn{9}{|c|}{ Depression $(296.2,296.3,296.5,296.6,305.8,311$, v790, 290.13) } \\
\hline All & 174(6.05) & 13085 & 13.3 & $650(11.42)$ & 39350 & 16.52 & $1.24(1.05-1.47)^{*}$ & $0.78(0.61-1.01)$ \\
\hline $18-39$ & $31(8.52)$ & 1901 & 16.31 & $128(13.54)$ & 7189 & 17.8 & $1.09(0.74-1.62)$ & $0.76(0.42-1.38)$ \\
\hline $40-64$ & $86(5.93)$ & 6923 & 12.42 & $382(11.25)$ & 23900 & 15.98 & $1.29(1.02-1.63)^{*}$ & $0.73(0.52-1.04)$ \\
\hline$\geq 65$ & $57(5.37)$ & 4261 & 13.38 & $140(10.36)$ & 8260 & 16.95 & $1.27(0.93-1.72)$ & $0.89(0.54-1.48)$ \\
\hline \multicolumn{9}{|c|}{ Sicca syndrome $(370.33,710.2)$} \\
\hline All & $78(2.71)$ & 13085 & 5.96 & $423(7.43)$ & 39350 & 10.75 & $1.80(1.42-2.30)^{* * *}$ & $1.27(0.86-1.89)$ \\
\hline $18-39$ & $4(1.10)$ & 1901 & 2.1 & $42(4.44)$ & 7189 & 5.84 & $2.78(1.00-7.74)$ & - \\
\hline $40-64$ & $40(2.76)$ & 6923 & 5.78 & $266(7.84)$ & 23900 & 11.13 & $1.93(1.38-2.69)^{* * *}$ & $1.14(0.67-1.94)$ \\
\hline$\geq 65$ & $34(3.20)$ & 4261 & 7.98 & 115(8.51) & 8260 & 13.92 & $1.75(1.19-2.56)^{* *}$ & $1.71(0.93-3.15)$ \\
\hline
\end{tabular}

$\mathrm{a}_{I R}$ incidence rate, per 1000 person-years, IRR incidence rate ratio

${ }^{*}:<0.05 ;{ }^{* *}:<0.01 ;{ }^{* * *} p<0.001$

${ }^{b}$ Hazard Ratio adjusted for times of outpatient and inpatient visit, Charlson comorbidity index score and type of service used - only herbal medicine, only acupuncture and combination of them

Table 4 The distribution of TCM and non-TCM user by major disease categories /diagnosis in patients with hepatitis C

\begin{tabular}{|c|c|c|c|c|c|}
\hline \multirow[t]{2}{*}{ Disease (ICD-9-CM) } & \multicolumn{2}{|c|}{ Non-TCM user $(N=2876)$} & \multicolumn{2}{|c|}{ TCM user $(N=5691)$} & \multirow[t]{2}{*}{$p$ value } \\
\hline & $n$ & $\%$ & $n$ & $\%$ & \\
\hline Infectious and parasitic disease (001-139) & 2862 & 99.51 & 5673 & 99.68 & 0.2218 \\
\hline Neoplasms (140-239) & 1289 & 44.82 & 3121 & 54.84 & $<0.0001$ \\
\hline Malignant(140-208) & 824 & 28.65 & 1491 & 26.20 & 0.0158 \\
\hline Benign (210-229) & 597 & 20.76 & 2152 & 37.81 & $<0.0001$ \\
\hline $\begin{array}{l}\text { Endocrine, nutritional and metabolic disease and immunity } \\
\text { disorder (240-279) }\end{array}$ & 1863 & 64.78 & 4133 & 72.62 & $<0.0001$ \\
\hline Blood and blood-forming organs (280-289) & 843 & 29.31 & 1876 & 32.96 & 0.0006 \\
\hline Mental disorder (290-319) & 1206 & 41.93 & 3360 & 59.04 & $<0.0001$ \\
\hline Nervous system (320-389) & 2087 & 72.57 & 5120 & 89.97 & $<0.0001$ \\
\hline Circulatory system (390-459) & 2123 & 73.82 & 4410 & 77.49 & 0.0002 \\
\hline Respiratory system (460-519) & 2548 & 88.60 & 5573 & 97.93 & $<0.0001$ \\
\hline Digestive system (520-579) & 2797 & 97.25 & 5669 & 99.61 & $<0.0001$ \\
\hline Genitourinary system (580-629) & 1760 & 61.20 & 4485 & 78.81 & $<0.0001$ \\
\hline $\begin{array}{l}\text { Complications of pregnancy, childbirth and the } \\
\text { puerperium (630-676) }\end{array}$ & 19 & 0.66 & 178 & 3.13 & $<0.0001$ \\
\hline Skin and subcutaneous tissue (680-709) & 1958 & 68.08 & 4830 & 84.87 & $<0.0001$ \\
\hline Musculoskeletal system and connective tissue (710-739) & 2091 & 72.71 & 5343 & 93.89 & $<0.0001$ \\
\hline Congenital anomalies (740-759) & 126 & 4.38 & 511 & 8.98 & $<0.0001$ \\
\hline Certain conditions originating in the perinatal period (760-779) & 13 & 0.45 & 43 & 0.76 & 0.0997 \\
\hline Symptoms, signs and ill-defined conditions (780-799) & 2569 & 89.33 & 5575 & 97.96 & $<0.0001$ \\
\hline Injury and poisoning (800-999) & 1997 & 69.44 & 5166 & 90.77 & $<0.0001$ \\
\hline
\end{tabular}


Table 5 Most common Chinese herbs and formula prescribed for patients with hepatitis $C$

\begin{tabular}{|c|c|c|c|c|c|}
\hline $\begin{array}{l}\text { Prescription name } \\
\text { (in Chinese) }\end{array}$ & Ingredients & Therapeutic action and Indication & $\begin{array}{l}\text { Number of } \\
\text { person-days }\end{array}$ & $\begin{array}{l}\text { Average daily } \\
\text { dose }(\mathrm{g})\end{array}$ & $\begin{array}{l}\text { Average duration for } \\
\text { prescription (days) }\end{array}$ \\
\hline \multicolumn{6}{|l|}{ Single herb } \\
\hline Dan-shen & Salvia miltiorrhiza Bunge & $\begin{array}{l}\text { H \& E: Activate blood and resolve stasis } \\
\text { anti-fibrosis, antihepatocarcinoma, anti-diabetic, } \\
\text { lipid-lowering }\end{array}$ & 59252 & 2.5 & 10.1 \\
\hline Yan-hu-suo & Corydalis yanhusuo & $\begin{array}{l}\text { H \& E: Activate blood, promote flow of qi, and } \\
\text { alleviate pain } \\
\text { Also used in peptic ulcer }\end{array}$ & 41875 & 3 & 7.1 \\
\hline Huang-qin & Scutellaria baicalensis Georgi & $\begin{array}{l}\text { H: Clear heat and drain fire } \\
\text { Anti-inflammation }\end{array}$ & 35273 & 2.2 & 8.1 \\
\hline Yin-chen-hao & Artemisia capillaris Thunb & $\begin{array}{l}\text { H: Excrete dampness and alleviate jaundice } \\
\text { Anti-fibrosis }\end{array}$ & 33357 & 2.4 & 10.1 \\
\hline Da-huang & Rheum officinale Baill & $\begin{array}{l}\text { E: Clear heat and drain fire } \\
\text { Anti-tumor }\end{array}$ & 32110 & 1.1 & 7.9 \\
\hline Huang-qi & Astragalus membranaceus & $\begin{array}{l}\text { H\&E: Qi-tonifying/ restore energy } \\
\text { Anti-cancer }\end{array}$ & 30086 & 2.1 & 8.8 \\
\hline Bei-mu & Fritillariae thunbergii Bulbus & $\begin{array}{l}\text { E: Clear heat and resolve phlegm } \\
\text { Also used in peptic ulcer and asthma }\end{array}$ & 29404 & 2.7 & 7.1 \\
\hline Ge-gen & Pueraria thomsonii Benth & $\begin{array}{l}\text { E: Release exterior and cure heat } \\
\text { Also used in ischemic heart disease }\end{array}$ & 29143 & 2 & 7.4 \\
\hline Ye-jiao-teng & Polygonum multiflorum Thunb. & $\begin{array}{l}\text { E: Nourish heart and induce tranquilization } \\
\text { Also used in menopausal syndrome }\end{array}$ & 28559 & 3.3 & 8.7 \\
\hline Hai-piao-xiao & Sepiella maindronide Rochebrune & E: Restrain acidity and alleviate pain & 27940 & 2.5 & 8.4 \\
\hline Formulae & & Also used in peptic ulcer & & & \\
\hline Jia-wei-xiao-yao-san & $\begin{array}{l}\text { Glycyrrhiza uralensis Fisch. Angelica sinensis, Atractylodes } \\
\text { macrocephala, Bupleurum chinense, Gardenia jasminoides, } \\
\text { Mentha haplocalyx, Paeonia lactiflora, Paeonia suffruticosa, } \\
\text { Poria cocos, Zingiber officinale }\end{array}$ & $\begin{array}{l}\text { H \& E: Harmonize liver and release spleen; } \\
\text { Also used in thyroid disorders }\end{array}$ & 88124 & 7.5 & 9.3 \\
\hline Xiao-chai-hu-tang & $\begin{array}{l}\text { Bupleurum chinense, Scutellaria baicalensis Georgi, Pinellia } \\
\text { ternata (Thunb.) Makino, Panax ginseng C.A. Mey, } \\
\text { Glycyrrhiza uralensis Fisch, Zingiber officinale, Zizyphus jujuba }\end{array}$ & $\begin{array}{l}\text { H: Regulate exterior and interior Qi activity by } \\
\text { balancing between yin and yang; } \\
\text { Antihepatocarcinoma }\end{array}$ & 39837 & 6.7 & 7.9 \\
\hline Long-dan-xie-gan-tang & $\begin{array}{l}\text { Gentiana scabra Bge, Scutellaria baicalensis Georgi, Gardenia } \\
\text { jasminoides Ellis, Alisma orientalis, Akebia quinata (Houtt.) } \\
\text { Decne., Plantago asiatica L., Angelica sinensis, Rehmannia } \\
\text { glutinosa (Gaert.) Libosch., Bupleurum chinense, Glycyrrhiza } \\
\text { uralensis Fisch. }\end{array}$ & $\begin{array}{l}\text { H: Purge fire in the liver and gallbladder, clear } \\
\text { away damp-heat in the lower burner; } \\
\text { Antiinflammation }\end{array}$ & 36293 & 11.3 & 7.9 \\
\hline
\end{tabular}


Table 5 Most common Chinese herbs and formula prescribed for patients with hepatitis C (Continued)

\begin{tabular}{|c|c|c|c|c|c|}
\hline Shu-jing-huo-xue-tang & $\begin{array}{l}\text { Angelica sinensis, Paeonia lactiflora Pall, Glycyrrhiza uralensis } \\
\text { Fisch, Rehmannia glutinosa (Gaert.) Libosch, Atractylodes } \\
\text { lancea, Achyranthes bidentata Blume, Citrus tangerina } \\
\text { pericarpium, Citrus reticulata Blanco, Prunus persica (L.) } \\
\text { Batsch, Clematis chinensis Osbeck, Ligusticum striatum } \\
\text { DC., Stephania tetrandra S. Moore, Notopterygium incisum, } \\
\text { Angelica dahurica (Fisch. ex Hoffm.) Benth, Gentiana scabra } \\
\text { Bge, Poria cocos, Zingiber officinale Rosc., }\end{array}$ & $\begin{array}{l}\text { E: Relax the channels and activate blood; } \\
\text { Also used in osteoarthritis and rheumatoid } \\
\text { arthritis }\end{array}$ & 32646 & 10.3 & 6.9 \\
\hline Xiang-sha-liu-jun-zi-tang & $\begin{array}{l}\text { Aquilaria sinensis, Amomum villosum Lour, Citrus reticulata } \\
\text { Blanco, Pinellia ternata (Thunb.), Panax ginseng C.A. Mey, } \\
\text { Poria cocos, Atractylodes macrocephala Koidz., Glycyrrhiza } \\
\text { uralensis Fisch. }\end{array}$ & $\begin{array}{l}\text { E: Tonify and replenish qi } \\
\text { Also used in functional dyspepsia and post-surgery } \\
\text { colon cancer patients }\end{array}$ & 28381 & 7.1 & 8.3 \\
\hline Gan-Iu-yin & $\begin{array}{l}\text { Rehmannia glutinosa, Asparagus cochinchinensis (Lour.) } \\
\text { Merr., Liriope spicata (Thunb.) Lour., Dendrobium nobile } \\
\text { Lindl., Artemisia capillaris Thunb, Scutellaria baicalensis } \\
\text { Georgi, Citrus aurantium L., Eriobotrya japonica (Thunb.) } \\
\text { Lindl., Glycyrrhiza uralensis Fisch, }\end{array}$ & $\begin{array}{l}\text { H\& E: Clear heat and nourish yin; } \\
\text { Also used in Sicca syndrome }\end{array}$ & 27563 & 6.1 & 7.6 \\
\hline Xue-fu-zhu-yu-tang & $\begin{array}{l}\text { Angelica sinensis (Oliv.) Diels, Ligusticum chuanxiong hort, } \\
\text { Paeonia anomala L., Prunus persica (L.), Batsch, Carthamus } \\
\text { tinctorius L., Rehmannia glutinosa (Gaert.) Libosch, Citrus } \\
\text { aurantium L., Bupleurum chinense DC., Glycyrrhiza uralensis } \\
\text { Fisch, Platycodon grandiflorum (Jaca.) A. DC., Achyranthes } \\
\text { bidentata Blume, }\end{array}$ & $\begin{array}{l}\text { E: Promote blood circulation to remove blood stasis } \\
\text { Also used in ischemic heart disease and hyperlipidemia. }\end{array}$ & 27493 & 6.6 & 8.1 \\
\hline Du-huo-ji-sheng-tang & $\begin{array}{l}\text { Angelica pubescens Maxim, Taxillus chinensis (DC.) Danser, } \\
\text { Eucommia ulmoides Oliver, Achyranthes bidentata Blume, } \\
\text { Asarum heterotropoides F. Schmidt, Gentiana macrophylla } \\
\text { Pall, Poria cocos, Cinnamomum cassia Presl, Saposhnikovia } \\
\text { divaricata (Turcz.) Schischk, Ligusticum striatum DC., Panax } \\
\text { ginseng C.A. Mey., Glycyrrhiza uralensis Fisch, Angelica sinensis, } \\
\text { Paeonia lactiflora Pall. }\end{array}$ & $\begin{array}{l}\text { E: Reinforce the liver and kidney and tonify qi and } \\
\text { blood; } \\
\text { Also used in fracture, osteoarthritis and rheumatoid } \\
\text { arthritis }\end{array}$ & 27388 & 11.1 & 8.2 \\
\hline Suan- zao-ren-tang & $\begin{array}{l}\text { Ligusticum striatum DC., Ziziphus jujuba Mill. Poria cocos, } \\
\text { Glycyrrhiza uralensis Fisch, Anemarrhena asphodeloides Bge }\end{array}$ & $\begin{array}{l}\text { E: Nourish blood to tranquilize the mind; } \\
\text { Also used in insomnia and depression }\end{array}$ & 26417 & 8 & 7.5 \\
\hline Ping-wei-san & $\begin{array}{l}\text { Citrus reticulata Blanco, Atractylodes lancea (Thunb.), } \\
\text { Magnolia officinalis Rehd, Glycyrrhiza uralensis Fisch }\end{array}$ & $\begin{array}{l}\text { E: Activate the flow of Qi and regulate the stomach } \\
\text { Also used in leukemia patients }\end{array}$ & 26083 & 7.2 & 7.6 \\
\hline
\end{tabular}

Note: $H$ hepatic action, $E$ extrahepatic action 
diagnostic records. Other $\mathrm{HCV}$ carriers without medical seeking behaviour would not be recorded in the national health insurance database.

Of the patients with $\mathrm{HC}, 66.4 \%$ had previously used TCM. The acceptance of TCM among patients with $\mathrm{HC}$ is much greater in Taiwan than in other countries $[11,12]$. In addition, approximately $16 \%$ of the patients visited TCM clinics more than six times per year (Table 2). This high visiting frequency might be explained by the fact that many of these patients had chronic illnesses that required long-term care and treatment. Moreover, unlike the predisposition towards acupuncture in Europe [23], herbal remedies have been widely used in Taiwanese patients with HC (52.4\%). People in Taiwan believe that TCM can adjust the constitution of the human body, allowing small doses of herbal remedies to remain safe and suitable for longterm use [24]. Furthermore, the insurance coverage for TCM treatments might play a significant role in the high TCM usage in Taiwan [25]. As for receiving the current standard treatment, the rate of treatment in both arms was low (14\% and $17 \%$ ) in our study. This result is consistent with a previous nationwide survey in Taiwan (13.7 \%) [26]. Although the anticipated treatment success rate is as high as $80 \%$ in Taiwan, only $8.1 \%$ of the population with $\mathrm{HC}$ achieved successful treatment. The major treatment barriers included fear of adverse effects, major disorders, ineligibility for insurance reimbursement, and lack of awareness of therapy.

Comparing the hazard ratio of comorbidities between TCM users and non-TCM users, the TCM users tended to have a lower risk $(0.4-0.6$ times $)$ of hepatic diseases, suggesting a negative association of hepatitis B, liver cirrhosis, and alcoholic liver disease with TCM usage. One possible explanation for this finding is that the use of TCM might have a protective effect on liver diseases. Another explanation is that patients with impaired liver function would avoid herbal medications to prevent disease progression. The causal relationship warrants further research in the future. However, thyroid diseases, rheumatoid arthritis, osteoarthritis, and sicca syndrome were extrahepatic syndromes that had higher incidence rates in TCM users in Taiwan (Table 4). After adjusting for time of visit, Charlson comorbidity index score, and type of TCM service used, the hazard ratio of these comorbidities became less than 1 and was non-significant. This means the TCM-seeking behaviour is associated with extrahepatic diseases rather than hepatitis $C$. This finding might be attributed to the side effects of interferon-based antiviral therapy and the lack of satisfaction with the current conventional therapies [27].

To date, the NHIRD has collected diagnosis data via ICD-9-CM codes, which do not classify TCM syndromes or diagnosis. However, TCM prescriptions including formula or herbs are recorded in the NHIRD. By analysing the prescription patterns, we could obtain the possible TCM syndromes and indications for subjects with HC (Table 5). In our database, the most commonly used formulas and single herbs for $\mathrm{HC}$ were categorized into hepatic or extrahepatic based on their therapeutic action and clinical indications. Jia-WeiXiao-Yao-San, the most commonly used formula, has demonstrated pleiotropic effects in patients with $\mathrm{HC}$, including anti-hepatic fibrosis [28], anti-hepatic cancer [29], anti-depressant [30, 31], and anti-hyperthyroidism effects [32]. However, it is unclear whether it has antiviral effects on $\mathrm{HCV}$, and future investigations on this subject are warranted. Dan-Shen (Salvia miltiorrhiza), the most commonly used single herb, also has multiple hepatoprotective and extrahepatic effects, such as antihepatic fibrosis [33], anti-hepatic cancer [34, 35], antidiabetic [36], and lipid-lowering [37] effects, but no apparent antiviral effects. Other commonly used prescriptions had similar multi-target effects, which implied that TCM physicians used these prescriptions to prevent disease progression or to relieve relevant extrahepatic syndromes rather than to eradicate HCV.

The present study had some limitations. First, this study did not include therapies that were not covered by the NHI, such as newly antiviral agents or folk medicines [38], which were purchased directly from TCM herbal pharmacies. Consequently, the TCM utilization rates might have been underestimated. However, because only licensed TCM physicians can be reimbursed by the NHI system, the quality of the diagnoses and treatments in the NHIRD were ensured. Second, safety data in this retrospective study are lacking, and thus we cannot evaluate the safety of TCM. Third, our study only examined ambulatory visits to TCM or non-TCM clinics. Our results for visits to Western medical clinics, including inpatient services and emergency department visits, basically concurred with a previous study of outpatient visits [39]. TCM inpatient services, which mostly included hospital-based healthcare for senile populations, were not that popular and therefore only represented a small proportion of the TCM services received by $\mathrm{HC}$ patients.

\section{Conclusion}

In summary, we conducted a nationwide, populationbased study on the use of TCM in patients with HC based on one randomly selected cohort in 2010 from the NHIRD healthcare claims data in Taiwan. It is that more than $60 \%$ of the TCM users were female and that the utilization of TCM increased with age and peaked in the age group of those $40-64$ years old. Patients who had more extrahepatic diseases and were taking more 
antiviral agents tended to visit TCM clinics. Jia-WeiXiao-Yao-San and Dan-Shen (Salvia miltiorrhiza) were the most commonly used formula and single herb, with 88,124 person-days and 59,252 person-days, respectively. The high prevalence and distinct usage patterns of TCM in the Taiwanese HC population warrant more substantial, high-quality and/or well-designed clinical trials of TCM use.

\section{Abbreviations}

CAM: Complementary and alternative medicine; HC: Hepatitis C; HCV: Hepatitis C virus; HR: Hazard ratio; ICD-9-CM: International Classification of Disease, 9th Revision, Clinical Modification; NCCIH: National Centre for Complementary and Integrative Health; NHI: National Health Insurance; NHIRD: National Health Insurance Research Database; TCM: Traditional Chinese Medicine; WHO: World Health Organization

\section{Acknowledgements}

This study is based in part on data from the National Health Insurance Research Database provided by the National Health Insurance Administration, Department of Health and managed by National Health Research Institutes. The interpretation and conclusions contained herein do not represent those of National Health Insurance Administration, Department of Health or National Health Research Institutes.

\section{Funding}

This study is supported by Taipei City Government under the research plan in 2015; China Medical University under the Aim for Top University Plan of the Ministry of Education, Taiwan. This study is also supported in part by Taiwan Ministry of Health and Welfare Clinical Trial and Research Center of Excellence (MOHW105-TDU-B-212-133019), China Medical University Hospital, Academia Sinica Taiwan Biobank Stroke Biosignature Project (BM10501010037), NRPB Stroke Clinical Trial Consortium (MOST 104-2325-B-039 -005), Tseng-Lien Lin Foundation, Taichung, Taiwan, Taiwan Brain Disease Foundation, Taipei, Taiwan, and Katsuzo and Kiyo Aoshima Memorial Funds, Japan.

\section{Availability of data and materials}

All data are deposited in a properly managed public repository. In this study, we examined and analyzed datasets released from the NHIRD (http:// nhird.nhri.org.tw/en/index.html), which are maintained and managed by National Health Research Institutes (http: www.nhri.org.tw/), Taiwan. The use of NHIRD is limited to research purposes only. Applicants must follow the Computer-Processed Personal Data Protection Law (http://www.winklerpart ners.com/?p=987) and related regulations of National Health Insurance Administration and National Health Research Institutes, and an agreement must be signed by the applicant and his/her supervisor upon application submission. All applications are reviewed for approval of data release.

\section{Authors' contributions}

CYL, HRY and $\mathrm{CHH}$ conceptualized the study. CYL and JHC performed the statistical analysis. CYL, JYC, HRY, CHH, and JHC contributed to the interpretation of TCM data. CYL, JYC, and HRY drafted the manuscript. CYL, $H R Y$, and $\mathrm{CHH}$ finalized the manuscript. All authors read and approved the final manuscript.

\section{Competing interests}

The authors declare that they have no competing interest.

\section{Consent for publication}

Not applicable.

\section{Ethics approval and consent to participate}

All of the datasets from the NHIRD were encrypted and de-identified to protect enrollee privacy. It was not possible to identify individual patients by any means. The Research Ethics Committees of Taipei City Hospital (TCHIRB-10406112-E), and China Medical University and Hospital (CMUH104-REC2-115) approved this study.

\section{Author details}

${ }^{1}$ Institute of Traditional Medicine, School of Medicine, National Yang-Ming University, Taipei, Taiwan. ${ }^{2}$ Department of Chinese Medicine, Branch of Linsen and Chinese Medicine, Taipei City Hospital, Taipei, Taiwan. ${ }^{3}$ School of Traditional Chinese Medicine, College of Medicine, Chang Gung University, Taoyuan, Taiwan. ${ }^{4}$ Research Centre for Chinese Medicine \& Acupuncture, China Medical University, Taichung, Taiwan. ${ }^{5}$ Health Data Management Office, China Medical University Hospital, Taichung, Taiwan. ${ }^{6}$ Graduate Institute of Integrated Medicine, College of Chinese Medicine, China Medical University, Taichung, Taiwan. ${ }^{7}$ Department of Chinese Medicine, China Medical University Hospital, 2 Yude Rd, North District, Taichung 404, Taiwan. ${ }^{8} \mathrm{~S} c h o o l$ of Chinese Medicine, College of Chinese Medicine, China Medical University, Taichung, Taiwan.

Received: 15 February 2016 Accepted: 8 October 2016

Published online: 21 October 2016

\section{References}

1. Tindle HA, Davis RB, Phillips RS, Eisenberg DM. Trends in use of complementary and alternative medicine by US adults: 1997-2002. Altern Ther Health Med. 2005;11(1):42-9.

2. Kessler RC, Davis RB, Foster DF, Van Rompay MI, Walters EE, Wilkey SA, Kaptchuk TJ, Eisenberg DM. Long-term trends in the use of complementary and alternative medical therapies in the United States. Ann Intern Med. 2001;135(4):262-8.

3. Organization WH. WHO traditional medicine strategy: 2014-2023. Geneva: Switzerland WHO press; 2013.

4. Seo HJ, Baek SM, Kim SG, Kim TH, Choi SM. Prevalence of complementary and alternative medicine use in a community-based population in South Korea: a systematic review. Complement Ther Med. 2013;21(3):260-71.

5. Rosen HR. Clinical practice. Chronic hepatitis C infection. N Engl J Med. 2011;364(25):2429-38.

6. van der Meer AJ, Wedemeyer H, Feld JJ, Hansen BE, Manns MP, Zeuzem S, Janssen $\mathrm{HL}$. Is there sufficient evidence to recommend antiviral therapy in hepatitis C? J Hepatol. 2014;60(1):191-6.

7. Modi AA, Wright EC, Seeff LB. Complementary and alternative medicine (CAM) for the treatment of chronic hepatitis B and C: a review. Antivir Ther. 2007;12(3):285-95

8. Metcalfe A, Williams J, McChesney J, Patten SB, Jette N. Use of complementary and alternative medicine by those with a chronic disease and the general population-results of a national population based survey. BMC Complement Altern Med. 2010;10:58

9. Saydah SH, Eberhardt MS. Use of complementary and alternative medicine among adults with chronic diseases: United States. J Altern Complement Med. 2002;12(8):805-12.

10. Robinson A, McGrail MR. Disclosure of CAM use to medical practitioners: a review of qualitative and quantitative studies. Complement Ther Med. 2004:12(2-3):90-8.

11. Richmond JA, Bailey DE, Patel K, Jezsik JA, Muir A, Lin JR, Chow SC, Uzarski D, McHutchison JG. The use of complementary and alternative medicine by patients with chronic hepatitis C. Complement Ther Clin Pract. 2010;16(3):124-31.

12. Ferrucci LM, Bell BP, Dhotre KB, Manos MM, Terrault NA, Zaman A, Murphy RC, Vanness GR, Thomas AR, Bialek SR, et al. Complementary and alternative medicine use in chronic liver disease patients. J Clin Gastroenterol. 2010;44(2):e40-45.

13. Gilmour J, Harrison C, Asadi L, Cohen MH, Vohra S. Informed consent: advising patients and parents about complementary and alternative medicine therapies. Pediatrics. 2011;128 Suppl 4:S187-192.

14. Park HL, Lee HS, Shin BC, Liu JP, Shang Q, Yamashita H, Lim B. Traditional medicine in china, Korea, and Japan: a brief introduction and comparison. Evid Based Complement Altern Med. 2012;2012:429103.

15. Chen FP, Chen TJ, Kung YY, Chen YC, Chou LF, Chen FJ, Hwang SJ. Use frequency of traditional Chinese medicine in Taiwan. BMC Health Serv Res. 2007;7:26

16. Chao TH, Fu PK, Chang CH, Chang SN, Chiahung Mao F, Lin CH. Prescription patterns of Chinese herbal products for post-surgery colon cancer patients in Taiwan. J Ethnopharmacol. 2014;155(1):702-8.

17. Liao YH, Lin CC, Li TC, Lin JG. Utilization pattern of traditional Chinese medicine for liver cancer patients in Taiwan. BMC Complement Altern Med. 2012;12:146 
18. Shih WT, Yang YH, Chen PC. Prescription patterns of chinese herbal products for osteoporosis in taiwan: a population-based study. Evid Based Complement Alternat Med. 2012;2012:752837.

19. Huang CY, Tsai YT, Lai JN, Hsu FL. Prescription pattern of chinese herbal products for diabetes mellitus in taiwan: a population-based study. Evid Based Complement Alternat Med. 2013;2013:201329.

20. Huang TP, Liu PH, Lien AS, Yang SL, Chang HH, Yen HR. A nationwide population-based study of traditional Chinese medicine usage in children in Taiwan. Complement Ther Med. 2014;22(3):500-10.

21. Chen FP, Kung YY, Chen YC, Jong MS, Chen TJ, Chen FJ, Hwang SJ. Frequency and pattern of Chinese herbal medicine prescriptions for chronic hepatitis in Taiwan. J Ethnopharmacol. 2008;117(1):84-91.

22. Sievert W, Altraif I, Razavi HA, Abdo A, Ahmed EA, Alomair A, Amarapurkar D, Chen $\mathrm{CH}$, Dou X, El Khayat $\mathrm{H}$, et al. A systematic review of hepatitis $\mathrm{C}$ virus epidemiology in Asia, Australia and Egypt. Liver Int. 2011;31 Suppl 2:61-80.

23. Coughlan BM, Thornton LM, Murphy N, Tait MA, Flanagan PC, Kenny-Walsh EE, O'Lorcain PP. The use of complementary and alternative medicine in an Irish cohort of people with an iatrogenic hepatitis $C$ infection: results from a health and lifestyle survey. Complement Ther Med. 2014;22(4):683-9.

24. Pu CY, Lan VM, Lan CF, Lang HC. The determinants of traditional Chinese medicine and acupuncture utilization for cancer patients with simultaneous conventional treatment. Eur J Cancer Care. 2008;17(4):340-9.

25. Wolsko PM, Eisenberg DM, Davis RB, Ettner SL, Phillips RS. Insurance coverage, medical conditions, and visits to alternative medicine providers: results of a national survey. Arch Intern Med. 2002;162(3):281-7.

26. Yu ML, Yeh ML, Tsai PC, Huang Cl, Huang JF, Huang CF, Hsieh MH, Liang $P C$, Lin YH, Hsieh MY, et al. Huge gap between clinical efficacy and community effectiveness in the treatment of chronic hepatitis $\mathrm{C}: \mathrm{a}$ nationwide survey in Taiwan. Medicine. 2015;94(13):e690.

27. Fallahi P, Ferrari SM, Politti U, Giuggioli D, Ferri C, Antonelli A. Autoimmune and neoplastic thyroid diseases associated with hepatitis $C$ chronic infection. Int J Endocrinol. 2014;2014:935131.

28. Chien SC, Chang WC, Lin PH, Chang WP, Hsu SC, Chang JC, Wu YC, Pei JK, Lin CH. A Chinese herbal medicine, jia-wei-xiao-yao-san, prevents dimethylnitrosamine-induced hepatic fibrosis in rats. TheScientificWorldJOURNAL. 2014;2014:217525.

29. Liao YH, Lin CC, Lai HC, Chiang JH, Lin JG, Li TC. Adjunctive traditional Chinese medicine therapy improves survival of liver cancer patients. Liver Int. 2015;35(12):2595-602.

30. Chen HY, Huang BS, Lin YH, Su IH, Yang SH, Chen JL, Huang JW, Chen YC Identifying Chinese herbal medicine for premenstrual syndrome: implications from a nationwide database. BMC Complement Altern Med. 2014;14:206.

31. Park HJ, Shim HS, Chung SY, Lee TH, Shim I. Soyo-san reduces depressivelike behavior and proinflammatory cytokines in ovariectomized female rats. BMC Complement Altern Med. 2014;14:34.

32. Chang CC, Huang ST. Is Traditional Chinese medicine effective for reducing hyperthyroidism? J Altern Complement Med. 2010;16(11):1217-20.

33. Parajuli DR, Zhao YZ, Jin H, Chi JH, Li SY, Kim YC, Sohn DH, Lee SH. Antifibrotic effect of PF2401-SF, a standardized fraction of Salvia miltiorrhiza, in thioacetamide-induced experimental rats liver fibrosis. Arch Pharm Res. 2015;38(4):549-55.

34. Hu X, Rui W, Wu C, He S, Jiang J, Zhang X, Yang Y. Compound Astragalus and Salvia miltiorrhiza extracts suppress hepatocarcinogenesis by modulating transforming growth factor-beta/Smad signaling. J Gastroenterol Hepatol. 2014;29(6):1284-91.

35. Rui W, Xie L, Liu X, He S, Wu C, Zhang X, Zhang L, Yang Y. Compound Astragalus and Salvia miltiorrhiza extract suppresses hepatocellular carcinoma progression by inhibiting fibrosis and PAl-1 mRNA transcription. J Ethnopharmacol. 2014;151(1):198-209.

36. Lee AL, Chen BC, Mou CH, Sun MF, Yen HR. Association of traditional Chinese medicine therapy and the risk of vascular complications in patients with type ii diabetes mellitus: a nationwide, retrospective, Taiwaneseregistry, cohort study. Medicine. 2016;95(3):e2536.

37. Chen J, Deng J, Zhang Y, Yang J, He Y, Fu W, Xing P, Wan H. Lipid-lowering effects of Danhong injection on hyperlipidemia rats. J Ethnopharmacol. 2014;154(2):437-42.

38. Shih SF, Lew-Ting CY, Chang HY, Kuo KN. Insurance covered and noncovered complementary and alternative medicine utilisation among adults in Taiwan. Soc Sci Med. 2008;67(7):1183-9.

39. Liao P, Ku M, Lue K, Sun H. Respiratory tract infection is the major cause of the ambulatory visits in children. Ital J Pediatr. 2011;37:43.

\section{Submit your next manuscript to BioMed Central and we will help you at every step:}

- We accept pre-submission inquiries

- Our selector tool helps you to find the most relevant journal

- We provide round the clock customer support

- Convenient online submission

- Thorough peer review

- Inclusion in PubMed and all major indexing services

- Maximum visibility for your research

Submit your manuscript at www.biomedcentral.com/submit 